\title{
Discussion on Expression Ways of Core Elements for Scientific Articles
}

\author{
Shu-hui WANG, Yan-ping NIU,Cai-juan SHANG, Sheng-feng WANG, and \\ Fei-fei SHANG
}

Department of Scientific Research, Academy of Armored Force Engineering, P.R. China, 100072

\begin{abstract}
Keywords: scientific articles, expression way, writing method, three-line table
Abstract. Both introduction and conclusion are important links of a scientific article, and their related successful description is a crucial factor in quality of the article. This paper is intended to analyze and summarize the key principles needing to be noticed in writing of introduction and conclusion, and discuss their respective main points to be followed. In addition, it sums up how to lay out the statistics diagrams/tables in a standardizing and skillful way in scientific articles, stating application points and analyzing improper expression ways.
\end{abstract}

\section{Introduction}

The scientific article is mainly used to describe the researches in all fields, and it is very different from literary works in the writing way. Emphasis should be laid on scientificness and preciseness in writing of the scientific article. As the authors of scientific articles are mostly researchers, so they consider fewer factors such as writing skills in their writings. In editing of Articles, some manuscripts were found very excellent in innovativeness and practicability, but authors' novel views couldn't be presented reasonably and accurately in their writing. This certainly would hinder the readability of periodicals as well as the propaganda and popularization of scientific information involved. This paper will analyze the matters needing attention in writing of introduction and conclusion, and sum up standard skills in preparing common diagrams and tables of scientific articles.

\section{Writing Requirements for Introduction}

The introduction, (also known as preface, foreword or overview), is an important link as the beginning of a scientific article ${ }^{[1,2]}$. In general, it is required to be compact in structure and concise in discussion for fear of an effect of the secondary superseding the primary. Its main contents generally include background and purpose, required grounds and current situations for the research; the summary of former work and study in relevant fields; the relation of the research and former studies, research hotspots, existing problems and the author's research significance, to draw forth the main body of the Article for readers ${ }^{[3,4]}$.

Both compactness and conciseness are necessary principles in writing of the introduction in order better to expound the issues studied in articles and guide readers to read and understand the full text. For example, the author should come to the point and not making the rounds in writing; avoid long description on the historical origin, establishment and study process of the project involved; do not discuss too much commonsense well known by the peers or in textbooks; mark others' research results and fundamental principles mentioned necessarily only in reference quotations. In introduction, the work and viewpoints should be presented in a clear and concise way; the approval and result evaluation procedures for the project, as well as related contracts, documents and all evaluation conclusions are unnecessary to be presented. Due to its relative conciseness, the introduction should not be stated by paragraphing generally, and not be added with illustrations and tables as well as the deduction and proof of formulas.

The introduction determines the general ketnote and orientation of an article to a great extent. So, it should be reasonable and practical in its description, and appropriate and graceful in description of its research significance, avoiding use of such descriptions as "very high academic value", "filling up domestic and overseas blank" and "finding for the first time". The expressions of politeness such as "little talent and less learning", "limited level" and "giving advice or comments" should not be 
applied. In addition, it is noted that the introduction should not have the same contents as its abstract as well as not be the explanatory notes to the abstract. It should echo with the conclusion generally, that is, the issues set forth in the introduction should be replied in the conclusion but not the same as that of the conclusion.

\section{Writing Method for Diagrams and Tables}

The statistic analysis is an indispensable approach in scientific research, and it is involved in processing and description of data. Both statistic diagram and table are common tools in descriptive statistic analysis, and they are employed to describe data in a visual, intuitionistic, simple and clear way, so that the data to be described are straightforward as a whole ${ }^{[5,7]}$. As the two tools are can keep your writing clear and engaging, they are the first choice in description of data and statistic results. The text description may sometimes make the results confused and difficult to identify differences for enormous and tedious data. However, both diagram and table may be employed to state research results concisely and clearly. Based on significance of diagrams and tables, it seems more important to learn statistic method and diagram/table editing skills.

Basic Application Norms of Diagram / Table. In the process of editing and processing, the diagram editing and processing norms should be well known. Firstly, the diagram should be self-explanatory to indicate the specific purpose, that is to say, the readers can understand its meaning only by viewing the diagram, its title and legend without main body reading. This means the diagram is strong explanatory and illustrative in the manuscripts. In addition, any diagram which is dispensable should not be applied as less as possible in case of readers' load in reading.

Applications of Table and Three-line Table Columns. The table is an important expression form of book contents. Just as the diagram, the table should be self-explanatory, but it is required to be stricter than the diagram. Background condition, comparison precondition, application method or computing data and final result should be listed clearly and separately in the table, so that the readers can be clear at a glance. Due to its unambiguous logical contrast, the readers can understand the object, method, purpose and result of one study without main body reading. Similarly, the table should come after its text. Although the table is clear and visual, it is not the more the better applied in an article. It may be selected based on the content to be stated in editing and processing. Like the diagram application norms, the table should not be employed any more if the content can be stated in fewer words.

Table 1 Data statistics for trial up to standard

\begin{tabular}{lccc}
\hline & Up to standard & Not up to standard & Total \\
\hline Group A & 72 & 28 & 100 \\
Group B & 85 & 15 & 100 \\
\hline
\end{tabular}

The data in Table 1 can be described in fewer words, but the table is applied for a duplicate expression, which leads to content repetition and layout waste. Hence, it is more concise and clearer if simple data are described in word instead of table. For example, it is show as Table 1.

Norms and Application of the Three-line Table. What is applied currently in scientific books is the three-line table in general. In fact, there are all kinds of diagrams and tables in many books and texts, and some tables, like a checkerboard, have transverse, vertical and oblique lines; the table is designed with the transverse lines with the number of rows of words, and vertical lines with the number of columns. Such tables are very disordered, not reflecting the object, purpose and result of the study intuitionistically, and the layout seems out of order on a printed page. This may result in reading disorders as shown by Table 2 . 
Table 2 Polarity matching relation analysis

\begin{tabular}{|c|c|c|c|c|}
\hline $\begin{array}{c}\text { Wetting effect } \\
\text { materials }\end{array}$ & Polarity & $\begin{array}{c}\text { Optimum } \\
\text { wetting }\end{array}$ & $\begin{array}{c}\text { Medium } \\
\text { wetting }\end{array}$ & $\begin{array}{c}\text { Poorest } \\
\text { wetting }\end{array}$ \\
\hline $\begin{array}{c}\text { Monocrystalline } \\
\text { silicon }\end{array}$ & Nonpolarity & Acid & Alkane & Alcohol \\
\hline Platinum sheet & $\begin{array}{c}\text { Certain } \\
\text { polarity }\end{array}$ & Alkane & Acid & Alcohol \\
\hline Stainless steel & High polartiy & Alkane & Acid & Alcohol \\
\hline
\end{tabular}

Table 2 may be changed by the three-line form shown in Table 3.

Table 3 Polarity matching relation analysis

\begin{tabular}{|c|c|c|c|c|}
\hline materials & Polarity & $\begin{array}{c}\text { Optimum } \\
\text { wetting }\end{array}$ & $\begin{array}{c}\text { Medium } \\
\text { wetting }\end{array}$ & $\begin{array}{l}\text { Poorest } \\
\text { wetting }\end{array}$ \\
\hline $\begin{array}{l}\text { Monocrystalline } \\
\text { silicon }\end{array}$ & Nonpolarity & Acid & Alkane & Alcohol \\
\hline Platinum sheet & $\begin{array}{l}\text { Certain } \\
\text { polarity }\end{array}$ & Alkane & Acid & Alcohol \\
\hline Stainless steel & $\begin{array}{l}\text { High } \\
\text { polartiy }\end{array}$ & Alkane & Acid & Alcohol \\
\hline
\end{tabular}

This expression may better show a corresponding relation of table title and test content, so it is clearer in description.

\section{Writing Requirements for Conclusion}

Conclusion is the final summary of the entire article. It is not a necessary part of an article although the authors end their articles by conclusion, and convey their main intentions to readers in most scientific articles [8]. If the required conclusion couldn't be derived obviously in an article, the necessary discussion may be given instead of the conclusion.

The conclusion should not be drawn by repeating summaries simply in paragraphs of the main body. It should include the following aspects completely, accurately and briefly on the basis of statement and analysis phenomena and data from experiment or observation in the text:

(1) The principles and other regularities revealed in the results obtained from observation or experiment of research objects;

(2) The issues which are exceptions in research or difficult to be explained and solved in the article in question.

(3) Similarities and differences from the former studies published (including others and the author self).

(4) Significance and values of the Article involved in theory and reality.

(5) Suggestions of a further in-depth study on the project involved.

\section{Summary}

1) The introduction is the beginning of an article. Good current situation analysis, and introduction and presentation to research orientation are the keys to the success to a good scientific article. The introduction should be created by the principles of conciseness, clearness, seeking truth from facts, and echo with conclusion.

2) Table is an important link for the expression of scientific articles. Writing articles by the standard three-line table is conducive to expounding of viewpoints. However, it is more concise for the simpler articles written directly in word. 
3) The conclusion is the final summary of the entire article. It should be expounded completely, accurately and briefly based on the statement and analysis of phenomena and data obtained by experiment or observation in the main body.

\section{References}

[1]. Blanka Frydrychova Klimova. Procedia - Social and Behavioral Sciences, Vol. 186(2015), p.908-912

[2]. Susan Simons, Jim Bryant, Jeanne Stroh. Computers and Composition, Vol. 12(1995), p.161-170

[3]. Daniel Oppenheimer, Franklin Zaromb, James R. Pomerantz, z, et.al. Assessing Writing, Vol. 32(2017), p.12-27

[4]. Blanka Frydrychova Klimova. Procedia-Social and Behavioral Sciences, Vol. 93( 2013), p. $512-516$

[5]. Alister Cumming, Conttia Lai, Hyeyoon Cho. Journal of English for Academic Purposes, Vol. 23, (2016), p. 47-58

[6]. Anthony Becker. Assessing Writing, Vol. 29( 2016), p.15-24

[7]. Ali Jamalinesari, Farahnaz Rahimi, Hsbib Gowhary,et.al. Procedia - Social and Behavioral Sciences, Vol. 192(2015), p.116-123

[8]. David Bunton. Journal of English for Academic Purposes, Vol. 4(2005), p. 207-224 